\title{
Sistemas de Hipermedia Adaptativa en la Educación. Aplicación en la Disciplina de Sistemas y Tecnologías de la Información para el Contador
}

\author{
Autora: Inés María González Vidal \\ Centro de Estudios para el Perfeccionamiento de la Educación Superior, \\ Universidad de La Habana, Cuba. \\ gonzvidalefcf.uh. cu \\ Tutor: Lázaro Blanco Encinosa \\ Centro de Estudios de Técnicas de Dirección, \\ Universidad de La Habana, Cuba. \\ lazaroj@ceted.uh.cu
}

\begin{abstract}
This work is the result of a doctoral thesis; this is the introduction of an adaptive hypermedia system in the training process of Information systems for accountant. Part of a diagnosis of this technology, from these items, defines the procedures for incorporation via the Moodle platform; adaptation criteria for the design of courses are presented; and general pedagogical guidelines for the development of courses arise. Finally, we proceed to the development of an experimental application; to assess the functionality of research results are proposed.
\end{abstract}

Resumen. Este trabajo es el resultado de una tesis de doctorado, es la introducción de un Sistema de Hipermedia Adaptativo en el proceso de formación de los Sistemas de información para Contador. Parte del diagnóstico de esta tecnologia; se definen los procedimientos para su incorporación a través de la plataforma Moodle; se presentan los criterios de adaptación para el diseño de los cursos; y directrices pedagógicas generales para el desarrollo de cursos de adaptación. Por último, se procede a la elaboración de una aplicación experimental; para evaluar se propone la funcionalidad de los resultados de investigación.

\section{Introducción}

La aplicación de las Tecnologías de la Información y la Comunicaciones impactan en los ámbitos educativos convencionales, así como en las barreras temporales, espaciales, socioculturales y económicas Blanco Encinosa, L. J. (1999), reafirmando que la educación virtual es una tendencia muy importante para la pedagogía contemporánea Adell, J. y Castañeda, L. (2013).

En este ámbito, la personalización de la enseñanza y la aplicación de los sistemas de recomendación han ido ganando terreno como componentes esenciales en las aplicaciones educativas Bork (2001), tanto en la prestación de apoyo a la navegación, como en la reducción de la sobrecarga cognitiva.

Al respecto, Brusilovsky, P. (1996) plantea que: "La adaptación ha demostrado que puede ayudar al alumno a adquirir conocimientos de manera más rápida, mejorar los resultados de aprendizaje, reducir los gastos generales de navegación y fomentar la navegación no secuencial". 
En este marco, se propone el diseño de un Sistema de Hipermedia Adaptativo para la asignatura Sistemas de Información para el Contador, con un enfoque donde se requiere la inserción de contenidos diversos que fortalezcan el proceso de interdisciplinariedad y que procedan a la combinación de tecnologías y áreas diferentes del conocimiento en una asignatura integradora.

\section{Análisis del problema de la investigación}

La asignatura Sistemas de Información para el Contador se caracterizada por la poca estructuración del contenido y el alto grado de creatividad exigido de los alumnos. Estudios realizados mostraron que los alumnos presentaban insatisfacción con relación al diseño tradicional del curso, y un universo de interés muy disperso.

Desde sus inicios, esa situación comenzaba a mostrar una vía de solución donde la adaptabilidad, las tecnologías novedosas y la alta motivación fueran las características más relevantes. Consecuentemente, se avizoraba el uso de la hipermedia adaptativa como una posible respuesta.

Se procedió, a analizar los sistemas de hipermedia adaptativa, a partir de información revisada; se detectó que en Cuba existían poquísimas experiencias anteriores. A nivel global, tampoco se apreciaba masividad en sus aplicaciones, lo que hizo pensar en la novedad científica que justificara la investigación. La literatura científica mostraba un creciente interés en la comunidad de investigadores, pues las incursiones reportadas cada vez son mayores. Todo ello conllevo a la definición del siguiente problema científico:

¿Cómo diseñar un sistema que permitiera adaptar los contenidos de la asignatura Sistemas de Información para el Contador, a las necesidades e interés profesional del alumno, de forma tal que se elevara la calidad de la enseñanza y a la vez se incrementara el arsenal de técnicas, métodos y herramientas didácticas, puestas a disposición de los profesores?

\section{Objetivo}

Diseñar un sistema de hipermedia adaptativa que contribuya a incrementar el aprovechamiento, por parte de los alumnos, de los contenidos de la asignatura Sistemas de Información para el Contador.

\subsection{Objetivos específicos}

1. Analizar críticamente los fundamentos teóricos, metodológicos y tecnológicos, sobre los que se apoya el diseño y la introducción de un sistema de hipermedia adaptativa.

2. Caracterizar las regularidades presentes en la asignatura para permitir su impartición mediante un sistema de hipermedia adaptativa.

3. Definir las acciones, criterios generales de adaptación y procedimientos necesarios para elaborar un sistema de hipermedia adaptativa ajustado a la asignatura de referencia.

4. Introducir experimentalmente el sistema de hipermedia adaptativa en la Licenciatura en Contabilidad y Finanzas.

5. Estudiar los resultados de la introducción experimental para obtener conclusiones que pudiesen estructurarse en una metodología para el futuro diseño sistema de hipermedia adaptativa. 


\section{CBIE-LACLO 2015}

Anais dos Workshops do IV Congresso Brasileiro de Informática na Educação (CBIE 2015)

6. Valorar la pertinencia de la metodología diseñada, a través de la aplicación experimental.

\section{Novedad de la investigación}

Los aspectos más novedosos de la presente investigación se relacionan con el diseño y aplicación de un Sistema de Hipermedia Adaptativa en la asignatura Sistemas de Información para el Contador, por primera vez en Cuba y probablemente una de las primeras en el mundo. Por lo cual puede afirmarse que los aportes teóricos, metodológicos y prácticos adquieren una novedad destacable.

\section{Contribución de la investigación}

El estudio de diferentes métodos para reconocer y explotar las características del alumno, permitirá obtener con mayor eficacia y eficiencia los conocimientos. El desarrollo de diseños de instrucción acordes con las necesidades del alumno y las áreas de conocimiento más afines con sus intereses profesionales, constituye un reto permanente para los profesores, que así podrán apoyarse en este tipo de sistemas. En este sentido, esta tesis aporta un modesto grano de arena en las áreas teóricas y metodológicas.

La metodología propuesta ayudará a los docentes en la construcción de un curso basado en hipermedia adaptativa, para solucionar las posibles insuficiencias en la flexibilidad de la gestión del sistema de contenidos que se presenta a los alumnos adecuándolo de manera personalizada y acomodada a las características de cada alumno y a sus preferencias y necesidades profesionales. Además, se definen los criterios generales de adaptación de apoyo a los diseñadores del curso y se describe el procedimiento orientado a desarrolladores de herramientas de autor a través de la Plataforma Moodle.

El principal aporte práctico es el diseño de un prototipo de sistema de hipermedia adaptativa, aplicado y en funcionamiento; pero, por supuesto, perfectible, para ayudar al docente en los problemas que afectan específicamente los intereses y necesidades profesionales de los alumnos. Se simula un aula donde se presentan alumnos con diferentes características y se muestra la manera de formalizar el conocimiento acerca de la detección de los mencionados intereses y necesidades profesionales.

\section{Métodos de investigación}

Entre los principales métodos generales y teóricos empleados se encuentran el método histórico-lógico, que permitió estudiar el objeto en su evolución y desarrollo en el contexto universitario. También el enfoque sistémico, favorecedor de la orientación general para identificar los aspectos esenciales que incluye la hipermedia adaptativa, desde la perspectiva Moodle para integrar cada uno de sus componentes. Se empleó el método de análisis y síntesis en el estudio de los antecedentes para la utilización de la Plataforma Moodle.

En el nivel empírico se emplearon varios métodos, técnicas y herramientas. Para recoger los datos y tener en cuenta su validez y fiabilidad se utilizaron diferentes instrumentos: entrevistas, encuestas, observaciones y el análisis documental. Se realizó un estudio exploratorio a través de una aplicación experimental, orientado a la valoración de la pertinencia de las propuestas.

Se empleó un estudio de inferencia a través de una prueba de hipótesis no paramétrica Chi cuadrado para demostrar que no existe correlación lineal entre estas variables. Se aplicaron técnicas y métodos de análisis y diseño de sistemas informativos, para crear bases de datos importantes para el trabajo durante la investigación. 


\section{Hipermedia adaptativa y modelado del alumno}

Los sistemas de hipermedia adaptativa son herramientas que ofrecen al alumno recursos que facilitan su trabajo personalizado y cooperativo Brusilovsky, P. (1996). El modelado de las características del alumno es el elemento fundamental para que un sistema pueda proveer y resolver problemas de adaptabilidad o personalización, por tanto, es el componente fundamental de cualquier hipermedia adaptativa. Graf, S. K. (2006), enfatiza en la necesidad de conocer a los alumnos antes de que la adaptabilidad sea proporcionada.

Este trabajo considera que las preferencias profesionales pueden ser afectadas durante la experiencia educativa del alumno. Identificarlas es un paso esencialmente crítico para mejorar la comprensión de cómo mejorar el proceso de aprendizaje.

\section{Estudio diagnóstico de la disciplina Sistemas de Información para el Contador}

La disciplina Sistemas y Tecnologías de la información para el Contador asume como propósito a formación profesional integral del alumno, sobre la base de la actividad científica investigativa, relacionando los problemas de la institución y los del territorio, a partir del dominio y despliegue de sistemas informáticos y recursos tecnológicos disponibles. Tiene un carácter teórico práctico, fundamentada en las técnicas generales de informática y de elaboración y utilización de sistemas, tanto manuales como automatizados, en sus posibilidades y aplicaciones en la contabilidad, las finanzas, la auditoría, la economía y la gestión en general.

Se realizó análisis de la matriz DAFO simplificada se sintetizan las debilidades, amenazas, fortalezas y oportunidades del colectivo. El diagnóstico se elaboró con indicadores que enfatizan los principales problemas y logros de la disciplina STIC. En ella se posicionan las condiciones externas e internas (factores) que facilitan y potencian, y lastran u obstaculizan el logro de los objetivos. Estos factores emergen a partir del análisis de la información histórica de la disciplina.

\section{Procedimientos para la incorporación de hipermedia adaptativa, a través de la Plataforma Moodle orientada a desarrolladores de herramientas de autor}

Diseño del curso en Moodle de la asignatura pero incorporando características de hipermedia adaptativa: En el formulario de entrada es donde se realiza la captación de datos por categorías, las asociadas con el acceso al sistema, datos personales y una nueva categoría, "Enfoque de conocimiento", donde se recogen las características que serán adaptadas.

Definición de la estructura de Bases de datos: La información referente al sistema de bases de datos serán transparentes al profesor. Moodle brinda facilidades para personalizar la información referente a los alumnos, esta característica sirve para definir un modelo del alumno y, además, para sugerir posibles caminos que se deben seguir en función de este.

Arquitecturas adaptativas para Moodle vinculada a los intereses profesionales del alumno (ver figura 1): La siguiente propuesta permite la incorporación de la adaptación vinculada a los intereses profesionales del alumno. Se agregan un conjunto de herramientas inteligentes basadas en las técnicas de hipermedia adaptativa para el análisis de diferentes parámetros, además del análisis de reglas y de recomendaciones, para el envío de la información personalizada al alumno en función de sus intereses. 


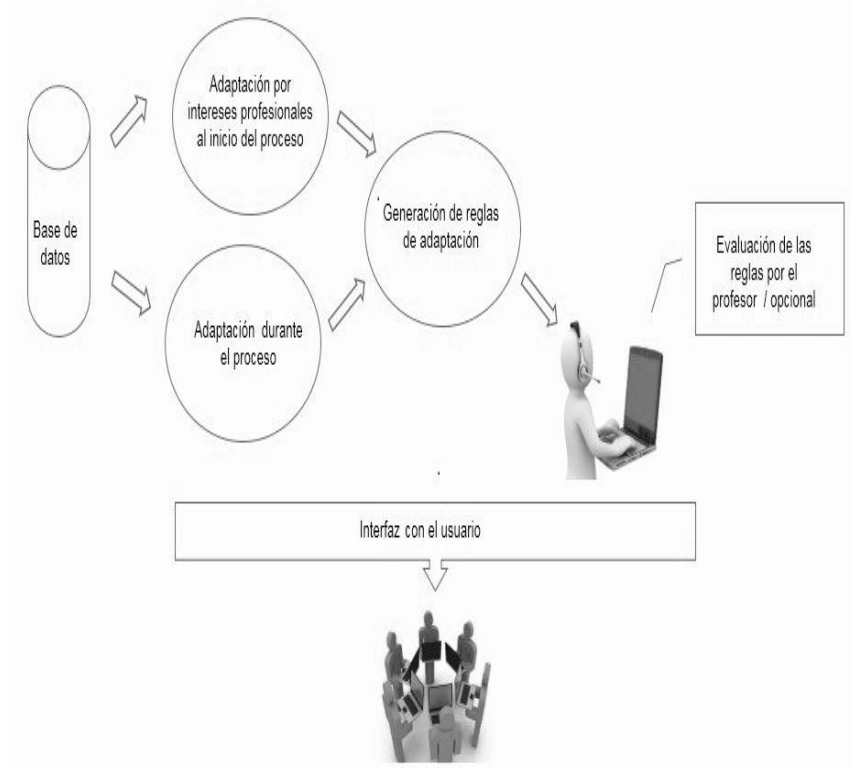

Figura 1. Arquitecturas adaptativas para la asignatura Sistemas de Información para el Contador. Fuente: Elaboración propia partir de las experiencias educativas en la asignatura.

Diseño de los instrumentos de recopilación de información: Con estos instrumentos de recopilación de información, el profesor obtiene información sobre la situación general del curso, el funcionamiento del sistema, las actividades y la forma en que son presentadas, realizadas y evaluadas, o sobre cualquier otro tema relacionado directo o indirectamente con el diseño del curso.

Generación de reglas de adaptación: Buscar una relación entre las acciones y los estados individuales de los alumnos, debe ser una proyección bien definida por el docente para posteriormente generar las reglas de adaptación. En la motivación y la satisfacción del alumno, participan los procesos afectivos, las tendencias y los procesos cognoscitivos; a su vez son, un reflejo de la realidad y por ello se debe buscar un mecanismo para medirlos y cuantificarlos, se reflejan en los resultados docentes.

\section{Criterios generales de adaptación que sirven de apoyo al diseñador del curso}

En un sistema de hipermedia, la adaptación afecta a los nodos y a los vínculos del hiperespacio, por ellos, es importante definir los elementos generales de adaptación que apoyan el trabajo del diseñador del curso adaptativo.

a) Criterios para la definición del dominio de aplicación. En un entorno educativo adaptativo, el modelo de dominio es la estructura de referencia para mantener un sistema actualizado de contenidos y objetivos por los que navegará el alumno en relación con la materia que se le presenta.

El dominio de aplicación incluye, además, anotaciones (metadatos), gráficas del dominio que modelan la estructura de los documentos (estructura del documento, relaciones jerárquicas, etc.), o gráficas de conocimiento que lo describen en colecciones de documentos.

El dominio de aplicación (ver figura 2) incluye, anotaciones (metadatos), gráficas del dominio que modelan la estructura de los documentos (estructura del documento, 
relaciones jerárquicas, etc.), o gráficas de conocimiento que lo describen en colecciones de documentos (ontologías del dominio).

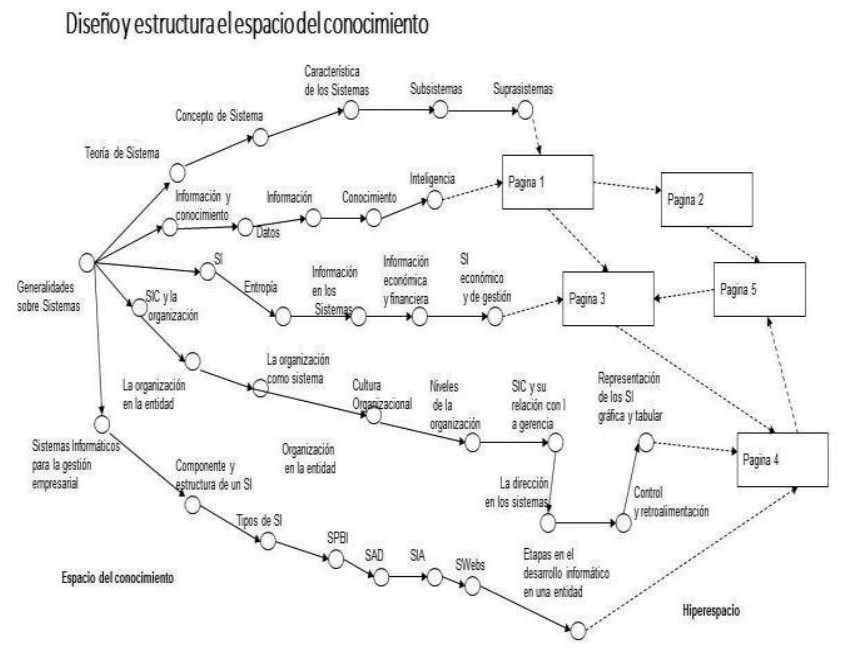

Figura 2. Representa un ejemplo de la estructura de conocimiento para un tema en particular. Fuente: Elaboración propia a partir de las experiencias educativas en la asignatura.

b) Criterios para la definición del modelo del alumno. El modelo del alumno contiene la información sobre a quién enseñar, sus preferencias, conocimiento, objetivos, historial, experiencias y conocimientos previos (tanto en la materia, como en el uso de sistemas de hipermedia).

c) Criterios para la definición del modelo del entorno. El sistema puede adaptarse a las características del soporte físico que utilice el usuario, pero mientras no se pidan requisitos previos de software o se pidan los mínimos, no será posible utilizar ciertas funciones del sistema.

d) Criterios para la definición del método de aplicación. Consiste en: 1) la adecuación de un programa o de un entorno adaptativo de trabajo, de acuerdo con las características del alumno. 2) la modificación de la forma de presentar los contenidos de los nodos o conceptos de información y los enlaces o relaciones entre ellos. 3) la adaptación de los soportes de navegación. Es la modificación de la presentación y comportamiento asociado en lo que se refiere a los enlaces.

e) Criterios para la definición del tipo de prerrequisitos. Para esta asignatura se analizan dos tipos: 1) prerrequisitos pedagógicos, que obedecen a las relaciones entre los contenidos y los aspectos relacionados con el aprendizaje. 2) prerrequisitos como mecanismo de ordenamiento, que tratan de establecer un orden parcial de las actividades que debe realizar el alumno.

f) Criterios para la definición del conjunto de objetivos de aprendizaje. Es la capacidad de construir un modelo de objetivos o metas educativas. Permite a un profesor asignar diferentes objetivos educativos a los diferentes alumnos que trabajan con el mismo sistema.

g) Criterios para definir la forma de adaptar la presentación de contenidos. En este caso. Explicaciones comparativas, variantes explicativas o ajuste del texto, generación de nodos completos: en este caso.

\section{Metodología orientada a docentes para la construcción de un curso utilizando hipermedia adaptativa}


CBIE-LACLO 2015

Anais dos Workshops do IV Congresso Brasileiro de Informática na Educação (CBIE 2015)

A continuación se muestra la metodología a seguir por los docentes para la construcción de un curso adaptativo, se incluyen algunas actividades sugeridas y temas a investigar durante el proceso.

\section{Proceso de creación del curso adaptativo.}

1. El profesor que construye el curso es el encargado de montar y proporcionar toda la información tanto de contenido como de estructura necesaria para el curso.

2. Utilizar una herramienta de autor genérica o específica para facilitar esta tarea.

- Facilidad de uso de la herramienta.

- Facilidad para comprender el formalismo de representación.

- Facilidad para definir la estructura de un curso.

- "Amigabilidad" de la herramienta.

- Utilidad de poder incluir dependencias entre tareas.

- Utilidad de la facilidad que permite comprobar la consistencia del curso.

- Utilidad de la posibilidad de colaborar con otros diseñadores.

3. Crear distintas versiones de un mismo curso según el perfil del alumno. Y la utilidad de poder incluir dependencias entre tareas.

\section{Ejecución del curso.}

- Los alumnos utilizando un navegador Web se deben conectar al servidor Web donde se encuentra localizado el curso para poder realizarlo.

- Mientras los alumnos ejecutan el curso de forma transparente, se recoge información de utilización que se va a almacenar en el servidor en los distintos ficheros logs.

\section{Mantenimiento del curso.}

1. Utiliza como entrada los datos de uso del curso por los alumnos del sistema y aplica un algoritmo de minería sobre los datos para detectar posibles problemas.

2. Los resultados de este proceso se muestran al profesor en forma de recomendaciones para que modifique la estructura o el contenido del curso.

3. Utilidad de la generación dinámica de enlaces.

\section{Actividades sugeridas}

Describe con textos sencillos: modelo de alumno, modelo del dominio y modelos del entorno.

\section{Investigue}

¿Cómo será la valoración de los estudiantes al utilizar un sistema de aprendizaje con hipermedia adaptativa?

¿Cómo será la valoración de los estudiantes respecto a los contenidos aportados por el sistema?

¿Cómo será la valoración de los estudiantes respecto a las evaluaciones sobre los contenidos que le realizará el sistema?

¿Cómo será la valoración de los estudiantes respecto a su perfil, generado por el sistema?

¿Considerarán los estudiantes que el uso de la hipermedia adaptativa tiene un efecto positivo sobre su proceso de aprendizaje?

¿Cómo influirá el modelo sobre el proceso de aprendizaje de cada individuo? 


\section{CBIE-LACLO 2015}

Anais dos Workshops do IV Congresso Brasileiro de Informática na Educação (CBIE 2015)

\section{Análisis de los resultados}

En este epígrafe se integra la valoración de expertos, una experiencia educativa con las propuestas, un análisis cualitativo de su pertinencia; y cierra con un estudio prospectivo de la aplicabilidad de los resultados en el contexto de referencia.

La utilización del criterio de expertos se sustenta en el principio de socialización y crítica de las propuestas elaboradas, en función de evaluar su pertinencia y contribuir a su optimización y perfeccionamiento, a partir de los criterios de un grupo de especialistas competentes en el área. Estos expertos son seleccionados a través de los procedimientos operacionales del método (criterio de expertos aplicación del método Delhi).Para ello, se procedió a la determinación de un grupo de 30 posibles expertos, se consultan especialistas reconocidos por sus resultados en el área de tecnología educativa. El procesamiento estadístico de los datos obtenidos indicó que el coeficiente de competencia $(\mathrm{K})$ es alto en 20 de los posibles expertos, al resultar mayor e igual a 0,8 . El resto, al manifestarse su coeficiente de competencia durante la evaluación, en los niveles Medio y Bajo, se desestimó. Como indica la información presentada en el trabajo doctorado, se consideraron como expertos, 20 del total de los encuestados; los que se caracterizan por su elevada preparación científico-metodológica en el tema. El resultado definitivo de esta experiencia concluyó con el logro de propuestas más coherentes y racionales, que responden con mayor exactitud a las exigencias actuales de la disciplina y la proyección en sus ámbitos de la hipermedia adaptativa.

Se realizó una valoración a partir de las opiniones de los docentes respecto a la propuesta. Algunos docentes, que no estaban familiarizados con el trabajo en plataformas virtuales mostraban su rechazo, pues se requería de un esfuerzo adicional; primero para emigrar a las plataformas virtuales de enseñanza y, posteriormente, para generar varias versiones del mismo contenido para el diseño del curso adaptativo. Otros profesores, se encontraban motivados con la nueva propuesta y argumentaban las ventajas de trabajar sobre Moodle, apoyados con la hipermedia adaptativa. Con el objetivo de confirmar o refutar la información obtenida por medio de las entrevistas y el análisis de documentos se realizó el diseño del sistema de observación, dirigida a partir de la metodología que aparece en Blanco Encinosa, L. J. (2008); el mismo concluye que no obstante a la resistencia de algunos, se logró que el $80.57 \%$ de las asignaturas de la disciplina migrasen a entornos virtuales y que, al menos tres asignaturas aplicaran experiencias adaptativas durante el proceso.

Se formalizó un análisis prospectivo de la aplicación de la hipermedia adaptativa en la disciplina, con el objetivo de moverse en el plano de un estudio de futuro, que ponga en manos de docentes y directivos, una valoración realista y crítica de las potencialidades que ofrecen los entornos adaptativos, en virtud del perfeccionamiento de las actividades educativas de las asignaturas en particular y de la carrera en general.

Se desarrolló una experiencia educativa para valorar el rendimiento escolar de los alumnos, si se aplican las técnicas de hipermedia adaptativa en función de su interés profesional del alumno, en el contexto de la Plataforma Moodle. Para ello se formaron dos grupos de trabajo, uno de control y otro experimental. Ambos grupos evidenciaban las mismas características en cuanto a la diversidad de procedencia de los alumnos, la heterogeneidad de sus miembros y la pluralidad de intereses profesionales. El grupo de control asistió a un curso virtual Moodle sin adaptación. En el grupo experimental, al inicio del proceso, se captó el interés profesional de los alumnos y, posteriormente, se crearon los grupos de trabajo por intereses afines (ver figura 3 ). 
CBIE-LACLO 2015

Anais dos Workshops do IV Congresso Brasileiro de Informática na Educação (CBIE 2015)

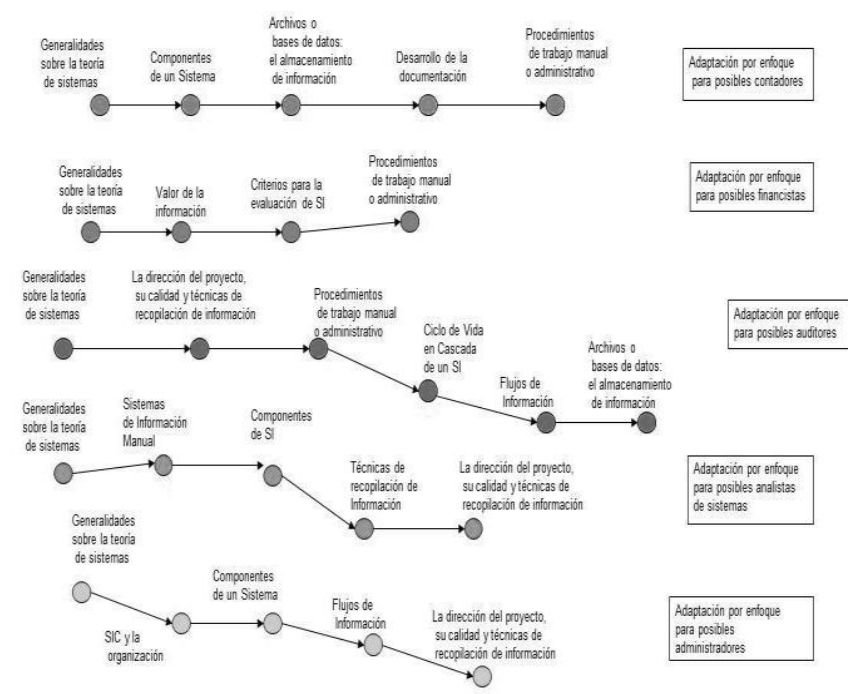

Figura 3. Adaptación de contenidos por interés profesional en la asignatura Sistemas de Información para el Contador. Fuente: Elaboración propia a partir de las experiencias educativas en la asignatura.

Los alumnos transitaron por contenidos diversos atendiendo a sus necesidades individuales y grupales. Al concluir, los alumnos del grupo experimental mostraron resultados docentes un $17 \%$ más estable, debido a que las propuestas de contenido se adaptaron a su interés profesional, sus expectativas y su motivación.

\section{Conclusiones}

La hipermedia adaptativa constituye un conjunto integrado de tecnologías, donde interactúan los subsistemas multimediales e hipertextuales, y los tutoriales inteligentes. Esta confluencia orienta su funcionalidad en torno a la personalización del proceso de enseñanza-aprendizaje en entornos virtuales, a través de las posibilidades de ajuste con respecto al modelo del alumno (sus necesidades e intereses); desde la óptica de los mecanismos de adaptación del hiperespacio, y en consonancia con el contenido educativo del entorno.

La adaptabilidad se refiere al relacionamiento del sistema de hipermedia adaptativa con respecto a las demandas del usuario (alumno), dimensión desde la cual se tienen en cuenta sus intereses profesionales, motivación, satisfacción y rendimiento, entre otros; como parámetros adaptativos a seguir en el diseño de entornos virtuales para la disciplina, a través de la introducción en este contexto de hipermedia adaptativa.

$\checkmark$ Desde el punto de vista formativo, la utilización de hipermedia adaptativa genera una dinámica colaborativa en el proceso de enseñanza-aprendizaje, que favorece la interacción de sus principales actores, y entre éstos y los materiales educativos virtuales contenidos en los entornos; además de proyectar una concepción metodológica orientada al incremento de los niveles de autonomía e independencia de los sujetos en formación, siempre con la consabida existencia de niveles de orientación y ayuda adecuados.

En la configuración de los sistemas de hipermedia adaptativa confluyen dos perspectivas de adaptación: la primera, en función de las necesidades e intereses profesionales de los alumnos al inicio del proceso, referida a la concepción inicial del sistema, donde se definen los modelos generales del entorno, del alumno, del tutor, de los contenidos y el de la actividad. La segunda, en virtud del requerimiento de 


\section{CBIE-LACLO 2015}

Anais dos Workshops do IV Congresso Brasileiro de Informática na Educação (CBIE 2015)

actualización de dicho sistema, la adaptación en tal sentido concierne a la progresiva correspondencia de los modelos definidos con las demandas de personalización del proceso.

$\checkmark$ Esta investigación contiene, entre sus principales resultados, una serie coherente y fundamentada de procedimientos garantes de la proyección de hipermedia adaptativa en el contexto de los entornos virtuales de enseñanza-aprendizaje que se desarrollan en Moodle. De igual modo, se definen, desarrollan y explicitan criterios de adaptación para la implementación de un sistema de hipermedia adaptativa en la disciplina Sistemas y Tecnologías para el Contador; y se plantean las orientaciones docentes para la construcción de un curso adaptativo, siguiendo los presupuestos antes mencionados.

La valoración y puesta en práctica de las propuestas contenidas en la investigación, dan fe de la pertinencia educativa de las mismas, al mostrar resultados que apuntan a la demostración de su funcionalidad; al grado de aceptación por parte de sus principales beneficiarios (docentes y alumnos); y a la validez de los resultados, en correspondencia con los requerimientos actuales del área socio-profesional de Contabilidad y Finanzas del territorio.

\section{Recomendaciones para trabajos futuros}

Continuar las investigaciones relacionadas con la hipermedia adaptativa, por estar en plena evaluación científica. Proceder a la capacitación general y sistemática a los profesores. Proceder al desarrollo de una herramienta de autor para adaptar contenidos, como uno de los elementos necesarios para dar continuidad a esta investigación. Propiciar la migración paulatina y creciente de la mayor parte de las asignaturas a plataformas virtuales e incluir criterios y técnicas de adaptación en las diferentes propuestas. Generalizar la propuesta de los Sistemas de Hipermedia Adaptativa a otras disciplinas de la carrera e incluso, a otras carreras de la educación superior, sobre la base de las experiencias obtenidas.

\section{Bibliografía}

Adell, J. \& Castañeda, L. (2013). El ecosistema pedagógico de los PLEs. En L. Castañeda y J. Adell (Eds.), Entornos Personales de Aprendizaje: Claves para el ecosistema educativo en red, (pp. 29-51) Alcoy: Marfil.

Blanco Encinosa, L. J. (1999). "La hiperuniversidad: el desafío a las universidades latinoamericanas". Revista Rencuentro. México pp.46-56.

Blanco Encinosa, L. J. (2008). "Sistemas de información para el economista y el contador". Editorial Félix Varela. La Habana. ISBN 978-959-07-0423-9

Bork: (2001). "What is needed for effective learning on the Internet".In: Educational Technology \& Society 4(3) SIN 1436-4522.

Brusilovsky, P. (1996). "Methods and techniques of adaptive hypermedia".School of Information Sciences.University of Pittsburg.

Brusilovsky, P. S. (1996). "An intelligent tutoring system on World Wide Web”.ELMART. In: Frasson, C., Gauthier, G., \&Lesgold, A. Berlin. Springer Verlag, 261-269. Intelligent Tutoring Systems (Lecture Notes in Computer Science, Vol. 1086).British Journal of EducationalTechnology (2003) I.

Graf, S. K. (2006). “An Approach for Detecting Learning Styles in Learning Management Systems".Proceedings of the International Conference on Advances Learning Technologies (ICALT 06), (págs. 161-163). 